\title{
Thermally Induced Bias Errors for a Fiber Coil with Practical Quadrupole Winding
}

\author{
Berk Osunluk $^{1,2}$, Serdar Ogut ${ }^{1,3}$, and Ekmel Ozbay ${ }^{2,3}$ \\ ${ }^{1}$ Department of Electrical and Electronics Engineering, Bilkent University, 06800, Ankara, Turkey \\ ${ }^{2}$ Aselsan Inc., Microelectronics, Guidance and Electro-optics Sector, 06011, Ankara, Turkey \\ ${ }^{3}$ Nanotechnology Research Center - NANOTAM, Bilkent University, 06800, Ankara, Turkey
}

\begin{abstract}
This paper presents an advanced thermal modeling of a fiber optic gyroscope (FOG) coil. We extended the current models to practical quadrupole winding. Model covers homogenization/dehomogenization parameters of fiber coil. A simulation environment is created by the Finite Element Method (FEM). Simulation environment is validated by comparing the results with laboratory FOG experiments.
\end{abstract}

Keywords-fiber optic gyroscope (FOG); fiber sensing coil; Shupe effect; elastooptical interactions; finite element model (FEM); quadrupole winding; homogenization; dehomogenization

\section{INTRODUCTION}

Fiber optic gyroscope (FOG) is a highly precise sensor for angular rotation measurement that is used for navigation, positioning, and stabilization. The precision limits of FOG are still being discussed widely. One of the main limits is the thermally induced rate error. Thermally induced rate error analysis is based on Shupe and elastooptic effects. Shupe effect describes the bias shift due to change in the temperature field through a fiber coil while the elastooptic effect is based on the change in the stress field [1], [2]. Quadrupole winding pattern is proposed in [3] to reduce the thermally induced rate errors.

In the literature, the quadrupole pattern is approached to be ideal symmetric through the coil. However, this symmetry is degraded due to practical necessities. In this letter, we built a model covering the properties of practical quadrupole pattern. Our model includes the turn length asymmetry of the fiber coil which results radial asymmetry. Secondly, practical pattern creates axial asymmetry increasing the thermally induced error. Furthermore, fiber coil is modeled as a transversely isotropic structure. We calculated the elastooptical interactions by homogenization/dehomogenization processes of the transversely isotropic structure.

We built a Finite Element Method (FEM) model in two stages: Detailed modeling of representative volume element (RVE) and modeling of whole fiber coil. Detailed fiber model defines the interactions inside RVE consisting fiber core, clad, coating and adhesive. Homogenization of the RVE and dehomogenization parameters are obtained by simulations of the detailed model. Second simulation provides the temperature and strain distributions by using the homogenized fiber coil model along with the spool and environment. A temperature profile which is ranging from $-40{ }^{\circ} \mathrm{C}$ up to $+60{ }^{\circ} \mathrm{C}$ is applied to the model so that fiber coil is exposed to temperature fluctuations. Under this profile, temperature and stress field distributions are obtained for each fiber turn and each time instant. Local strain fields inside the fiber core are calculated by dehomogenization process using the global stress and temperature fields. Time derivatives of local temperature and strain fields are used to calculate the Shupe and elastooptic errors, respectively.

Theoretical calculations and modeling is validated with experiments. Experiments are carried on three fiber coils having same design parameters. Exposed temperature profile ranges from $-40{ }^{\circ} \mathrm{C}$ up to $+60{ }^{\circ} \mathrm{C}$ under temperature rates between $\pm 0.2^{\circ} \mathrm{C} / \mathrm{min}$. We report that theoretical and experimental results are consistent.

\section{THEORY}

Thermal fluctuations create nonreciprocal phase shift between counterpropagating waves in the fiber coil. Nonreciprocal phase shift is defined by Shupe [1]. Combining his equation with Sagnac relation, bias error $\Omega_{S}(t)$ for a FOG can be written as:

$$
\Omega_{S}(t)=\frac{n}{L D}\left(\frac{\partial n}{\partial T}+n \alpha\right) \int_{0}^{L} \dot{T}(z, t)(L-2 z) d z
$$

where $L$ is the length of fiber, $D$ is the diameter of fiber coil, $n$ is the refractive index of fiber core, $\partial n / \partial T$ is the temperature coefficient of $n, \alpha$ is thermal expansion coefficient of fiber core, $\dot{T}(z, t)$ is the temperature field time derivative and $z$ is the fiber portion where the temperature fluctuates.

Reference [2] extends the analysis for elastooptic interactions in fiber coil. It is shown that a change of the stress fields results bias error, $\Omega_{E O}(t)$ in FOG output. Relation can be written as follows:

$$
\Omega_{E O}(t)=\frac{n}{L D} \int_{0}^{L}\left[A \dot{\varepsilon}_{z}(\mathrm{z}, \mathrm{t})-\mathrm{B} \dot{\varepsilon}_{r}(\mathrm{z}, \mathrm{t})\right](L-2 z) d z
$$

where $\dot{\varepsilon}_{z}$ and $\dot{\varepsilon}_{r}$ are the time derivatives of the axial and radial strain fields inside the fiber core, respectively.

$$
A=\mathrm{n}\left(1-\frac{n^{2}}{2} p_{12}\right), B=\frac{n^{3}}{2}\left(p_{11}+p_{12}\right), \text { where } p_{12} \text { and } p_{11}
$$
are the photoelastic coefficients of the fiber glass.

These two main bias error mechanisms are additive. So the total bias error is

$$
\Omega_{\mathrm{Total}}=\Omega_{\mathrm{EO}}+\Omega_{S}
$$




\section{MODELING OF FIBER COIL}

Fiber coil consists of fiber turns, potting material between the turns and a spool. Fiber itself consists of core, cladding and coating. Excluding the spool, RVE of the fiber coil structure is obtained for homogenization/dehomogenization [4], [5]. After obtaining the homogenized fiber coil model, FEM environment is built with spool. Global temperature and strain fields are obtained by FEM simulations. Lastly, dehomogenization procedure is carried. In this stage, global fields are mapped to local fields which are used for the calculation of bias error.

\section{A. Homogenization of Fiber Coil}

Fiber coil is an orthotropic and inhomogeneous structure. Structure is homogenized by defining RVE (Fig. 1) and calculating the composite material properties by using the boundary conditions. Calculated parameters for our design is given in Table I.

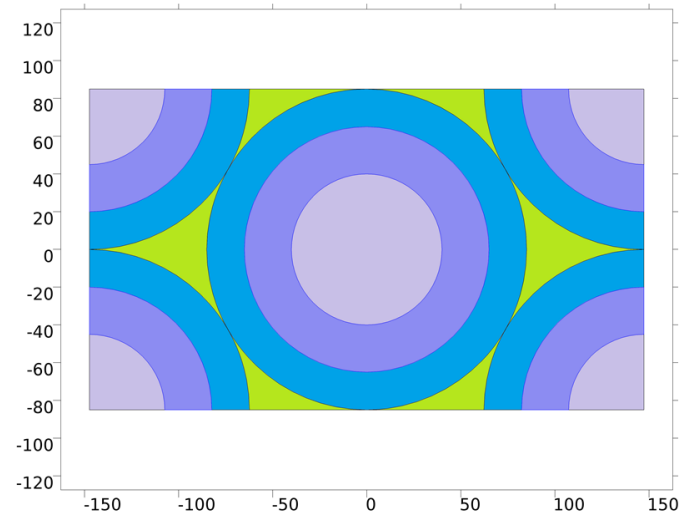

Fig. 1. Simulation of RVE with high resolution mesh. Fibers located orthocyclic manner with adhesive in between. All dimensions are in $\mu \mathrm{m}$.

TABLE I. COIL PARAMETERS OBTAINED BY HOMOGENIZATION

\begin{tabular}{|l|c|c|}
\hline \multirow{4}{*}{ Elastic Moduli } & $E_{z}(\mathrm{GPa})$ & 14.5 \\
\cline { 2 - 3 } & $E_{r}(\mathrm{MPa})$ & 95.2 \\
\cline { 2 - 3 } & $G_{z r}(\mathrm{MPa})$ & 24.1 \\
\hline \multirow{3}{*}{ Poisson's Ratio } & $v_{z r}$ & 0.392 \\
\cline { 2 - 3 } & $v_{r}$ & 0.979 \\
\cline { 2 - 3 } Thermal expansion coefficient & $v_{r z}$ & 0.003 \\
\cline { 2 - 3 } & $\alpha_{z}\left(\times 10^{-6} / K\right)$ & 3.36 \\
\hline \multirow{3}{*}{ Thermal conductivity } & $\alpha_{r}\left(\times 10^{-6} / K\right)$ & 193 \\
\hline & $k_{z}(\mathrm{~W} / \mathrm{mK})$ & 0.51 \\
\cline { 2 - 3 } & $k_{r}(\mathrm{~W} / \mathrm{mK})$ & 0.34 \\
\hline
\end{tabular}

\section{B. Obtaining Temperature and Macroscopic Strain Fields}

FEM simulation is built for obtaining the temperature and strain fields. FEM model consists of the homogenized fiber coil wounded on a spool, air surrounding the coil and the spool and heat source encapsulating the air (Fig. 2). Heat source provides a temperature profile ranging from $-40{ }^{\circ} \mathrm{C}$ to $60^{\circ} \mathrm{C}$. FEM simulation calculates the heat flow according to the material properties of spool and the fiber coil.

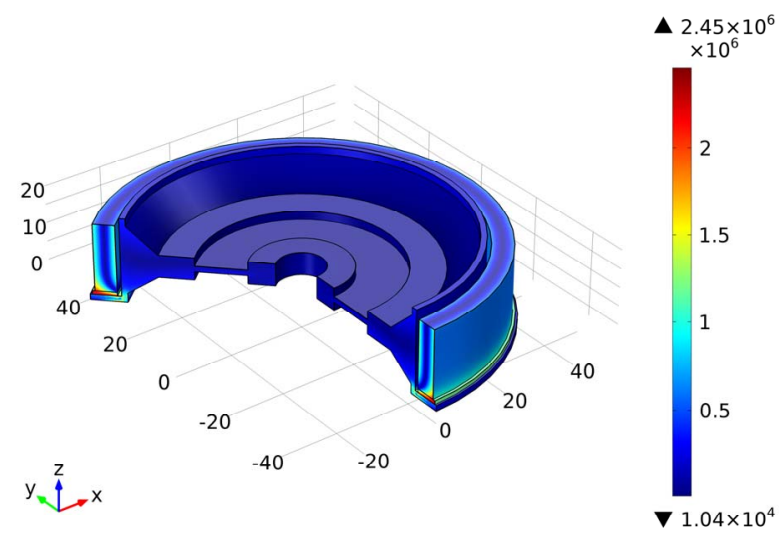

Fig. 2. FEM simulation of the fiber coil model.

\section{Dehomogenization of Fiber Coil}

FEM simulation outputs macroscopic temperature and strain fields. Strain fields inside the core are calculated by dehomogenization process. Dehomogenization of the global fields are just a transfer function, as follows:

$$
\left(\begin{array}{l}
\bar{\varepsilon}_{r, F} \\
\bar{\varepsilon}_{z, F}
\end{array}\right)=\mathrm{M}\left(\begin{array}{c}
\bar{\varepsilon}_{x x} \\
\bar{\varepsilon}_{y y} \\
\bar{\varepsilon}_{z z} \\
\Delta T
\end{array}\right)
$$

where $\bar{\varepsilon}_{x x}, \bar{\varepsilon}_{y y}, \bar{\varepsilon}_{z z}$ are the global strain fields obtained by FEM simulation in three dimensions and $\Delta T$ is the local temperature difference. $M$, the transformation matrix,

$$
M=\left[\begin{array}{cccc}
\mathrm{M}_{r x} & \mathrm{M}_{r y} & \mathrm{M}_{r z} & \mathrm{M}_{r T} \\
0 & 0 & 1 & 0
\end{array}\right]
$$

Transformation matrix elements are obtained along with homogenization process. Calculated matrix elements are given in Table II.

TABLE II. DEHOMOGENIZATION PARAMETERS

\begin{tabular}{|c|c|}
\hline $\mathrm{M}_{r x}$ & $2.74 \times 10^{-6}$ \\
\hline $\mathrm{M}_{r y}$ & $2.70 \times 10^{-6}$ \\
\hline $\mathrm{M}_{r z}$ & -0.17 \\
\hline $\mathrm{M}_{r T}$ & $9.98 \times 10^{-6} 1 / \mathrm{K}$ \\
\hline
\end{tabular}

\section{Bias Errors Calculation}

Microscopic strain and temperature fields are used in integrals given in (1) and (2), along with distance of each fiber turn from the fiber end point. Distance of each turn is calculated using the practical quadrupole cross section view (Fig. 3). 


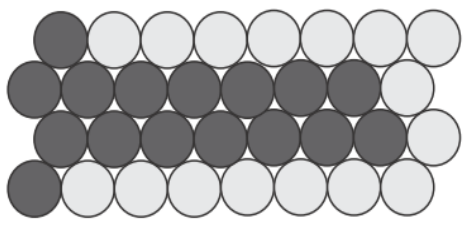

Fig. 3. Practical quadrupole pattern.

Practical quadrupole winding has some major differences from ideal quadrupole. Firstly, the length and diameter of turns differ in each layer of the fiber coil, which creates additional radial asymmetry. Secondly, first turn of each layer is wound either clockwise $(\mathrm{CW})$ or counter-clockwise $(\mathrm{CCW})$ and last turn vice versa. Therefore, fiber coil shows a figure with each side is either $\mathrm{CW}$ or $\mathrm{CCW}$ wound. This is a practical solution for passing the fiber from a layer to the next one. This second difference creates an axial asymmetry. We note that axial asymmetry is not negligible especially under thermal stress. Lastly, fiber turns are located in an orthocyclic manner, which mainly shape the RVE.

\section{SimUlation}

We built a coil model based on fiber coils produced for experiments. Design parameters of coils are given in Table III.

TABLE III. COIL PARAMETERS

\begin{tabular}{|l|c|}
\hline Fiber Length & $1101.57 \mathrm{~m}$ \\
\hline Number of winding layer & 36 \\
\hline Number of loop per layer & 106 \\
\hline Inner radius of the coil & $87.00 \mathrm{~mm}$ \\
\hline Outer radius of the coil & $97.65 \mathrm{~mm}$ \\
\hline Coil Height & $18.02 \mathrm{~mm}$ \\
\hline
\end{tabular}

Input temperature profile spans a range from $-40{ }^{\circ} \mathrm{C}$ to $+60{ }^{\circ} \mathrm{C}$ while the temperature is both increasing and decreasing. This profile reveals all temperature and temperature time derivative dependent errors in the interval.

Temperature versus time for each fiber turn along the fiber coil is given in Fig. 4. From the figure, it is seen that gradient along fiber turns is much slower than the derivative with respect to time. In other words, temperature is distributed along fiber coil faster than the temperature change. Also we note that elastooptical error is greater than the pure Shupe error. Furthermore, axial strains are dominant with respect to radial strains.

Microscopic strain fields obtained after dehomogenization are given in Fig. 5 and Fig. 6. The time derivatives of the fields are used in the calculation of the elastooptical bias error.

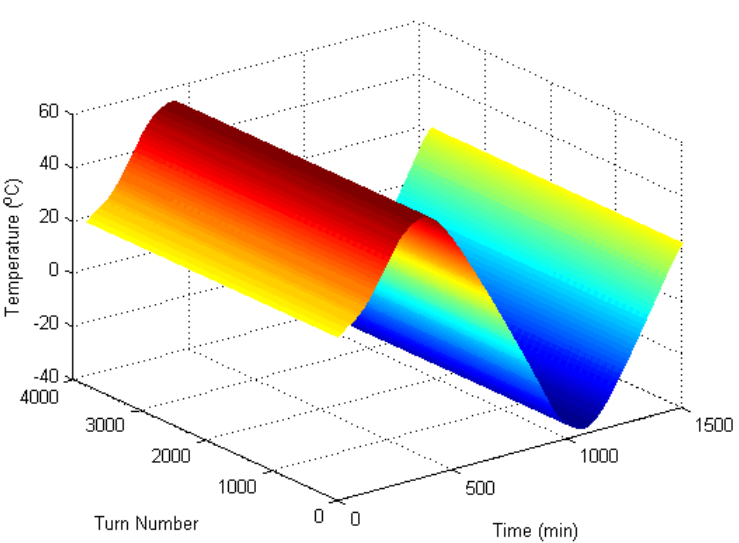

Fig. 4. Temperature distribution along fiber coil.

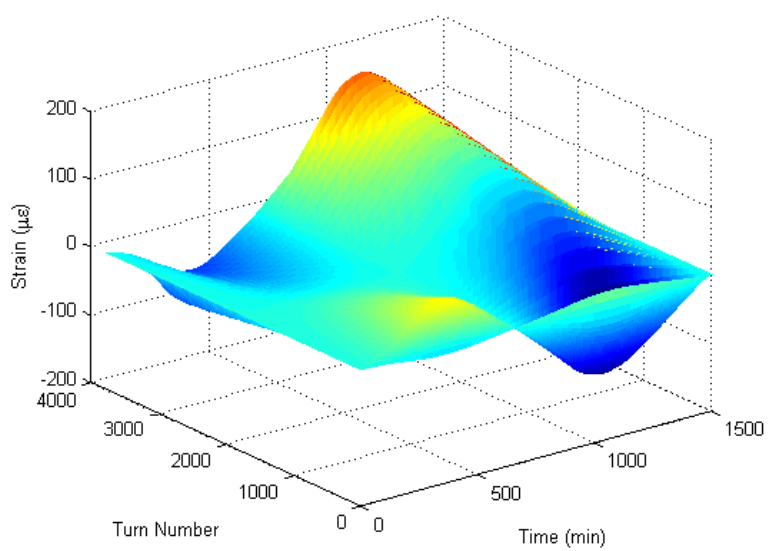

Fig. 5. Strain (radial) distribution along fiber coil.

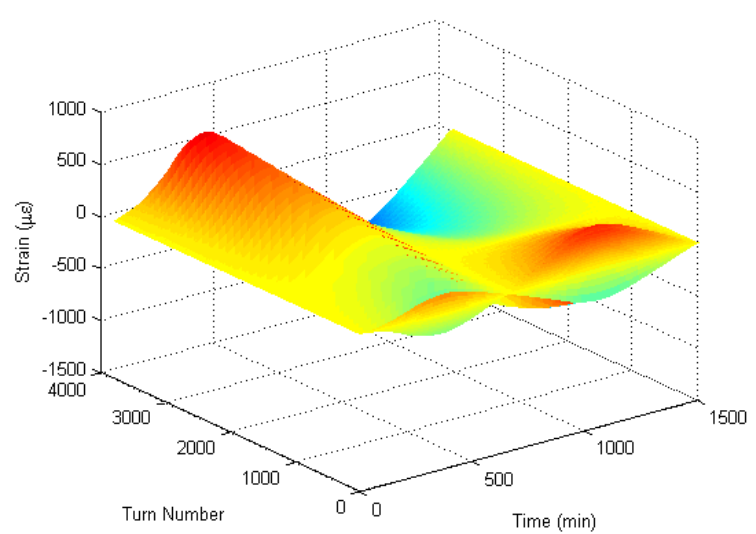

Fig. 6. Strain (axial) distribution along fiber coil. 


\section{EXPERIMENT}

Experiments are carried with three fiber coils having same design parameters as in simulations. Temperature sensors are mounted on each coil spool, representing the coil temperature. Temperature profile ranging from $-40{ }^{\circ} \mathrm{C}$ to $+60{ }^{\circ} \mathrm{C}$ is applied to fiber coils while temperature and rate measurement data are collected. Collected rate measurement is processed for eliminating the earth rotation and gyro noise. Collected FOG data and simulation results are plotted in Fig. 7. Calculated temperature sensitivity coefficients are given in Table IV.

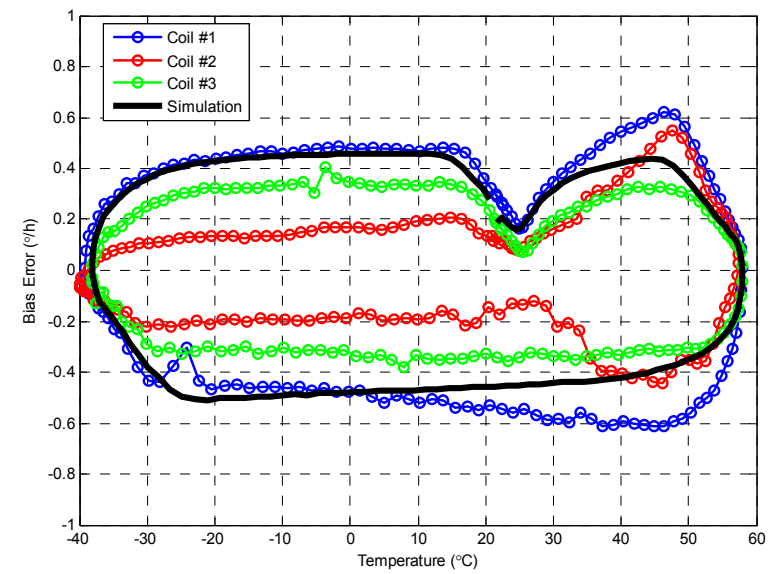

Fig. 7. Simulated and experimental bias error curves.

TABLE IV. SENSITIVITY COEFFICIENTS

\begin{tabular}{|l|c|}
\hline \multicolumn{1}{|c|}{ Coil No } & $\begin{array}{c}\text { Sensitivity Coefficient } \\
\left(\% / /{ }^{\circ} \mathbf{C} \text { min }\right)\end{array}$ \\
\hline Coil $\# 1$ & 3.01 \\
\hline Coil $\# 2$ & 1.39 \\
\hline Coil $\# 3$ & 1.98 \\
\hline Theoretical Model & 2.71 \\
\hline
\end{tabular}

Bias error characteristics for three fiber coils are consistent with theoretical model. Difference between the sensitivity coefficients of the coils could be a result of fiber tail length asymmetry during the production of coils. Also a change of the amount of the adhesive during the production could be another reason. It is also seen that sensitivity coefficient of Coil \#1 and Coil \#2 change for different temperatures. This phenomenon is thought to be related to the well-known racket effect.

\section{CONCLUSION}

In this letter, we built an advanced fiber coil model covering the Shupe effect, elastooptical interactions, orthotropic coil model and practical quadrupole winding pattern. We report experiments of three identically designed fiber coils and comparison of the results with simulation calculations. Experimental and simulation results are consistent.

\section{REFERENCES}

[1] D. M. Shupe, "Thermally induced non-reciprocity in the fiber optic interferometer," Applied Optics, vol. 19, no. 5, pp. 654-655, 1980.

[2] F. Mohr, "Bias error fiber optic gyroscopes due to elastooptic interactions in the sensor fiber," Proceedings of SPIE, vol. 5502, pp. 410-413, 2004

[3] N. J. Frigo, "Compensation of linear sources of non-reciprocity in Sagnac interferometers," Proceedings of SPIE, vol. 412, pp. 268-271, 1983.

[4] F. Schadt, "Physikalische modellierung und analyse thermisch und mechanisch bedingter messabweichungen in faseroptischen rotationssensoren", Magdeburg University, PhD Dissertation, 2012.

[5] S. Minakuchi, et. al., "Thermal strain in lightweight composite fiber-optic gyroscope for space application”, Journal of Lightwave Technology, vol. 33, no. 12, pp. 2658-2662, 2015. 